\title{
Topological Edge States at a Tilt Boundary in Gated Multilayer Graphene
}

\author{
Abolhassan Vaezi, ${ }^{1}$ Yufeng Liang, ${ }^{2}$ Darryl H. Ngai, ${ }^{1}$ Li Yang, ${ }^{2}$ and Eun-Ah Kim ${ }^{1}$ \\ ${ }^{1}$ Cornell University, Ithaca, New York 14853, USA \\ ${ }^{2}$ Washington University, St. Louis, Missouri 63130, USA
}

(Received 12 February 2013; revised manuscript received 3 May 2013; published 25 June 2013)

\begin{abstract}
Despite much interest in engineering new topological surface (edge) states using structural defects, such topological surface states have not been observed yet. We show that recently imaged tilt boundaries in gated multilayer graphene should support topologically protected gapless edge states. We approach the problem from two perspectives: the microscopic perspective of a tight-binding model and an $a b$ initio calculation on a bilayer, and the symmetry-protected topological (SPT) state perspective for a general multilayer. Hence, we establish the tilt-boundary edge states as the first concrete example of the edge states of symmetry-protected $\mathbb{Z}$-type SPT, protected by no-valley mixing, electron-number conservation, and time-reversal $T$ symmetries. Further, we discuss possible phase transitions between distinct SPTs upon symmetry changes. Combined with a recently imaged tilt-boundary network, our findings may explain the long-standing puzzle of subgap conductance in gated bilayer graphene. This proposal can be tested through future transport experiments on tilt boundaries. In particular, the tilt boundaries offer an opportunity for the in situ imaging of topological edge transport.
\end{abstract}

DOI: 10.1103/PhysRevX.3.021018

Subject Areas: Graphene, Materials Science, Topological Insulators

\section{INTRODUCTION}

Graphene has garnered interest from a broad spectrum of communities, ranging from those aiming at atomic-scale circuit devices to those searching for new topological phases. Both communities sought ways to gap the massless Dirac spectrum. The realization of a gate-induced band gap in the Bernal-stacked bilayer graphene [1] following the prediction in Ref. [2] brought the holy grail of the graphene-based transistor one step closer to reality. However, the subgap conductance measured by Oostinga et al. [1] with weak temperature dependence well below the optically measured gap as large as $250 \mathrm{meV}$ [3] introduced a new puzzle and obstacle: The gapped bilayer is not as insulating as it should be. Li et al. [4] proposed the ingap transport through the physical edge of the sample. However, such edge transport would disappear in the Corbino geometry of a clean sample. On the other hand, Yan and Fuhrer [5] observed two-dimensional variablerange-hopping-type temperature dependence for both the Corbino and the usual geometry. In this paper, we predict the existence of a topological gapless channel of transport along a recently imaged $A B-B A$ tilt-boundary network [6-8] that may solve the puzzle.

The predicted topological edge state holds the promise of the first realization of a topological surface (edge) state hosted by a structural topological defect. Although there has been much theoretical interest in topological gapless

Published by the American Physical Society under the terms of the Creative Commons Attribution 3.0 License. Further distribution of this work must maintain attribution to the author(s) and the published article's title, journal citation, and DOI. modes hosted by structural topological defects [9-11], no such topological gapless mode has been observed so far. The lattice dislocations in the three-dimensional crystals that were previously discussed occur deep in the sample that is not directly accessible. However, the tilt boundary of interest has recently been observed [6-8]. The tilt boundary is a structural topological line defect along which each neighboring layer is displaced by one interatomic spacing. Such a defect can occur because of the third dimension added by the stacking of the graphene layers; it forms a boundary between two inequivalent stacking structures frequently referred to as $A B$ and $B A$. Here, we show that the tilt boundaries host gapless modes of topological origin and form the first example of a naked structural defect hosting topological electronic states.

Topological aspects of gapped multilayer graphene have been previously discussed [12], and it was pointed out that they should exhibit the quantum valley Hall (QVH) effect with corresponding edge states. However, to this date, there has been no experimental detection of the proposed edge state $[4,13]$. Moreover, little is known about how the topological aspects of gapped multilayer graphene relate to topological insulators $[14,15]$. The idea of classifying different topological-insulator (superconductor) candidates based on symmetries [16] has played a key role in the field of topological insulators. In particular, the observation that additional symmetries such as the crystalline symmetries can enlarge the possibilities of topological phases $[17,18]$ has led to the discovery of threedimensional topological crystalline insulators [19]. We propose feasible experiments to detect topological edge states at naturally occurring tilt boundaries. At the same time, we make the first concrete application of the 
symmetry-protected topological (SPT) approach [17] for a two-dimensional (2D) system and study a large class of gapped graphene systems, placing the quantum valley Hall insulator in the larger context and predicting conditions for topological superconductors (TSCs).

The rest of the paper is organized as follows. In Sec. II, we show that an $A B-B A$ tilt boundary in gated bilayer graphene supports gapless edge states through explicit microscopic calculations. Specifically, we consider an abrupt boundary in a tight-binding model and then investigate the effect of strain using $a b$ initio calculation. In Sec. III, we show that these edge states are protected by no-valley mixing, electron-number conservation, and time-reversal (T) symmetries within the framework of SPT. Hence, we identify chirally stacked, gated $N$-layer graphene layers as time-reversal-symmetric $\mathbb{Z}$-type SPT. In Sec. IV, we discuss experimental implications. Finally, in Sec. V, we summarize the results and comment on practical implications.

\section{BILAYER TILT-BOUNDARY EDGE STATE}

Figures 1 and 2(a) show the tilt boundaries of interest in gapped Bernal-stacked bilayer graphene. In the case sketched, strain is concentrated at the tilt boundary with the top layer stretched by one interatomic spacing with respect to the bottom layer. For a general orientation, tilt boundaries can involve both strain and shear. As the tilt boundaries in layered graphene form a type of topological line defect in structure, they can be characterized using the tangent vector $\vec{t}$ and the Burger's vector $\vec{b}$. The tangent vector $\vec{t}$ points along the tilt boundary, which can point along any direction with respect to the Burger's vector $\vec{b}$. When the tilt boundary only involves strain, as in the case depicted in Figs. 1 and 2(a), the $\vec{b}$ is perpendicular to $\vec{t}$. In the opposite extreme limit of $\vec{b} \| \vec{t}$, shear is concentrated at the boundary. Independent of the angle between $\vec{b}$ and $\vec{t}$, the Burger's vector magnitude is the interatomic spacing, i.e., $|\vec{b}|=a$ for a bilayer system, as it is shown explicitly for the strain tilt boundary in Fig. 2(a). Since $|\vec{b}|$ is a fraction of the Bravis lattice primitive vector magnitude

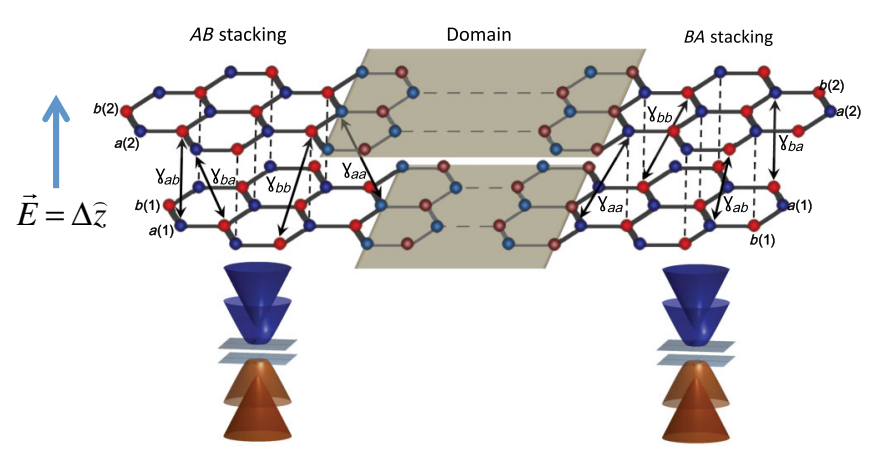

FIG. 1. A typical $A B-B A$ tilt boundary under strain. The blue (red) filled circles mark the $a(b)$ sublattice sites. $\gamma_{i j}$ represent hopping matrices for a tight-binding model.

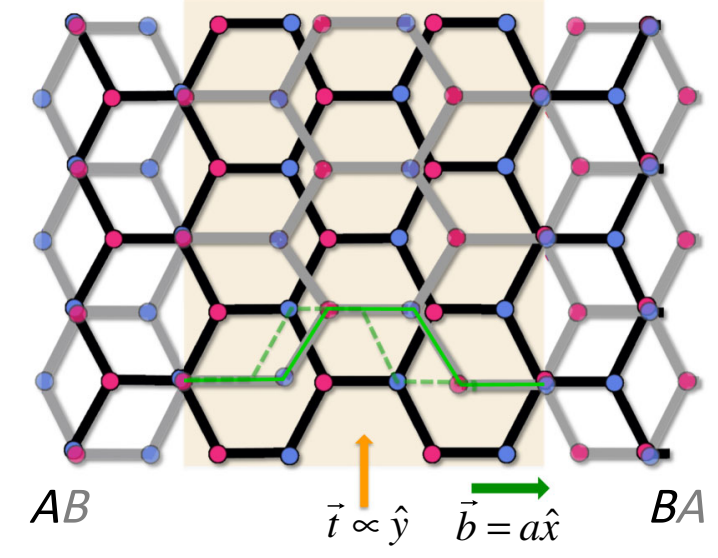

(a)

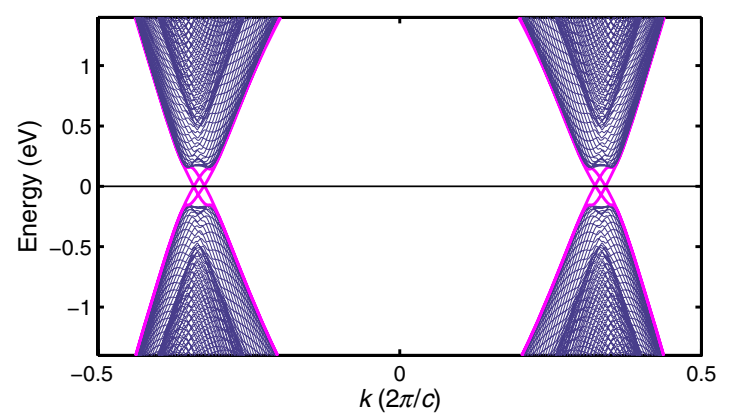

(b)

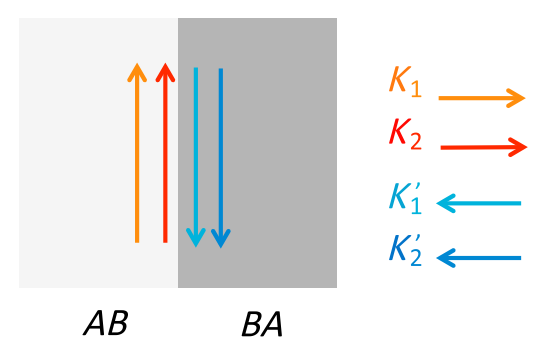

(c)

FIG. 2. A tight-binding study in the presence of abrupt $A B-B A$ tilt boundaries in gated bilayer graphene. (a) Schematic representation of the domain wall under strain. The grey (black) lines denote the upper (lower) layer, and solid blue (red) circles denote the $A(B)$ sublattice points. The tilt boundary along tangent vector $\vec{t} \| \hat{y}$ is shaded. The Burger's vector $\vec{b}=a \hat{x}$ (green arrow) for interatomic spacing $a$ accounts for the difference between the solid green line and the dashed green line. (b) The resulting band structure. The edge states are marked with magenta lines. (c) Schematics of valley-momentum-locked edge states at a tilt boundary.

$\sqrt{3} a$, the bilayer domain boundary is a partial dislocation from the quasi-two-dimensional view. In a general multilayer, a vertical array of these partial dislocations forms a tilt boundary. In typical samples, the domain wall separating the $A B$ - and $B A$-stacked domains has substantial width, spanning 5-20 nm, and the angle between $\vec{b}$ and $\vec{t}$ ranges between $0^{\circ}$ and $90^{\circ}[6]$. 
Figure 1 allows us to makes two important microscopic observations about tilt boundaries. (1) As the boundary requires a shift of one layer with respect to the other by one interatomic spacing along the bond direction, there are three natural directions for the tilt boundary to run for each fixed angle between $\vec{b}$ and $\vec{t}$. (2) The boundary is armchairshaped for $\vec{b} \| \vec{t}$ (pure shear), whereas it is zigzag for $\vec{b} \perp \vec{t}$ (pure strain case, shown in Fig. 1). Based on observation (1), we expect a given type of tilt boundary to possibly form a triangular network seen in experiments [6-8]. Observation (2), combined with earlier microscopic studies of boundary-condition effects on edge states in Ref. [20], implies that the electronic spectrum at tilt boundaries with $\vec{b} \| \vec{t}$ will be gapped though the gap magnitude and will be small when the tilt boundary is spread over a finite width.

In this section, we consider the electronic structure of tilt boundaries with $\vec{b} \perp \vec{t}$ and return to more a general case in Sec. IV. As it is shown schematically in Fig. 1, the bulk of each domain is gapped in the presence of interlayer hopping and the external electric field. The latter is important for breaking the inversion symmetry between the layers and gapping otherwise touching bands [1,2]. Below, we present two separate microscopic calculations of the $A B-B A$ tilt-boundary electronic structure for $\vec{b} \perp \vec{t}$, which shows gapless edge states.

\section{A. Tight-binding model}

We consider a tight-binding Hamiltonian with nearest-neighbor intra- and interlayer hopping. For the $A B$-stacking region [see Fig. 2(a)],

$$
\begin{aligned}
H_{A B}= & -t \sum_{i=1}^{2} \sum_{m, n} a_{m, n}^{(i)}{ }^{\dagger}\left(b_{m, n}^{(i)}+b_{m-1, n}^{(i)}+b_{m, n-1}^{(i)}\right) \\
& +\Delta \sum_{i=1}^{2} \sum_{m, n}(-1)^{i}\left(a_{m, n}^{(i)}{ }^{\dagger} a_{m, n}^{(i)}+b_{m, n}^{(i)}{ }^{\dagger} b_{m, n}^{(i)}\right) \\
& -t_{\perp} \sum_{m, n} a_{m, n}^{(1)}{ }^{\dagger} b_{m, n}^{(2)}+\text { H.c. },
\end{aligned}
$$

where $i=1,2$ is a layer index; $t$ and $t_{\perp}$ are intralayer and interlayer nearest-neighbor hoppings, respectively; and $\Delta$ is the chemical potential difference between two layers due to the gate voltage. $(m, n)$ labels the position of the two-site unit cell, with $a_{m, n}^{(i)}$ and $b_{m, n}^{(i)}$ annihilating electrons at the two sites of layer $i$. For the $B A$-stacked region, the only change is in the interlayer term with $\left(-t_{\perp} \sum_{m, n} b_{m, n}^{(1)} \dagger^{\dagger} a_{m, n}^{(2)}+\right.$ H.c. $)$ replacing the interlayer term in $H_{A B}$. As we address the effect of strain through $a b$ initio simulation, we focus here on a sharp tilt boundary, as shown in Fig. 2.

We plot the energy spectrum in Fig. 2(b) with the model parameters set to be $t=2.8 \mathrm{eV}, t_{\perp}=0.4 \mathrm{eV}$, and $\Delta=0.5 \mathrm{eV}$. The size of the system was 200 unit cells in each direction under the periodic boundary condition, with each domain spanning a 100-unit-cell width separated by two sharp tilt boundaries. From the spectra, it is clear that $K$ and $K^{\prime}$ valleys each have two edge states per spin. Further investigation of the wave function shows that rightand left-moving edge states associated with the given valley are spatially separated between the two edges: The edges offer valley filtering [see Fig. 2(c)].

\section{B. Ab initio simulation}

The electronic structure in a realistic tilt boundary will be affected by both the span over which the lattice structure transitions from $A B$ to $B A$ stacking and the strain concentrated at the tilt boundary. To address these issues, we further carry out first-principles calculation using densityfunctional theory (DFT) within the local-density approximation (LDA) [21,22]. We construct a periodic supercell with two tilt boundaries characterized by Burger's vectors identical to the ones considered within tight-binding calculation, but the domain wall is set to have finite width over which the 1-unit-cell mismatch is spread. We have tried several configurations with domains and tilt boundaries of different widths to find qualitatively similar results. In the rest of this paper, we focus on a representative example with a 3.1-nm-wide domain wall between 1.5-nm-wide domains. The choice of narrow width for the domains is due to the limitation in the simulation capability. To this 2D system, we then apply a slightly exaggerative perpendicular electric field of $5 \mathrm{~V} / \mathrm{nm}$. By relaxing the domain boundaries until the forces reach below $0.5 \mathrm{eV} / \mathrm{nm}$, we take the effect of both strain and width into account. We have tried several configurations with domains and tilt boundaries of different widths but otherwise similar settings to find qualitatively similar results.

The DFT-LDA simulation results are presented in Fig. 3, which confirms the existence of the gapless edge states predicted by the tight-binding model. The electronic structure along the extended direction of the edge is shown in Fig. 3(a), focusing on the region near the $K$ point. In this figure, two gapless 1D Dirac dispersions are clearly resolved from the gapped bulk spectra, with two distinct Dirac points $K_{1}$ and $K_{2}$ in the vicinity of the $K$ point. The strain concentrated at the tilt boundary causes energy splitting of the $K_{1}$ and $K_{2}$ states; the energy of the 1D Dirac points is increased by compressive strain and decreased by tensile strain. This energy splitting will become negligibly small in realistic tilt boundaries with much wider spans, as the strain will become smaller. Figure 3(a) shows that the existence of gapless edge states found in our tight-binding calculation is robust against long-range perturbations such as tilt-boundary width or subtle bond-length variation inside the domain wall, as well as the strain at the tilt boundary.

We now turn to the spatial distribution of the gapless edge states. For illustration, Fig. 3(b) shows the wave-function 


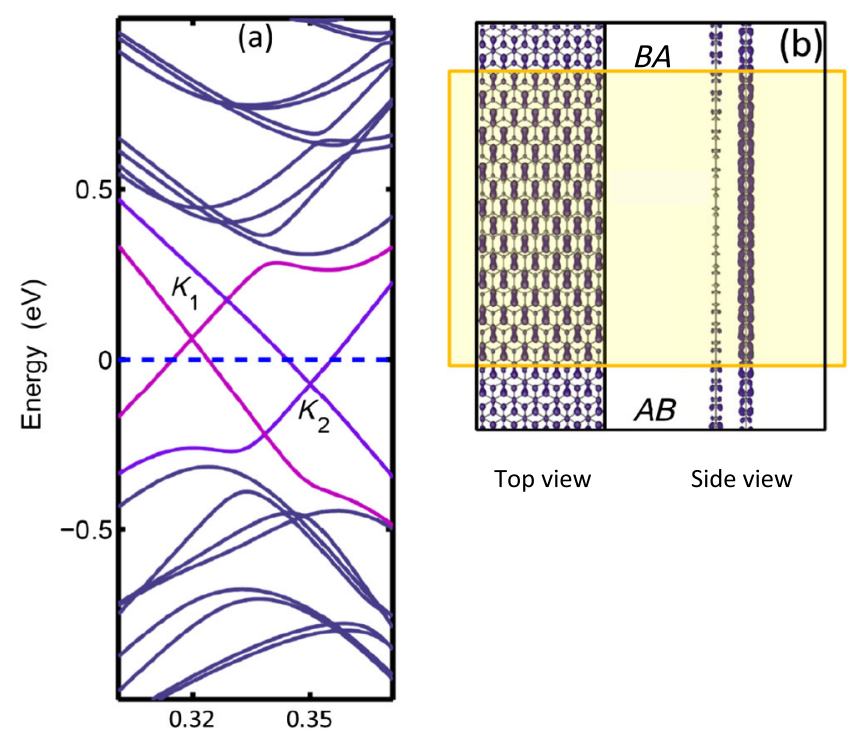

FIG. 3. (a) The energy bands of $A B-B A$ bilayer graphene connected to the two domain walls depicted in Fig. 2. The Fermi level is indicated with the dashed blue line, and the bands with the marked linear dispersion relation intersecting near the Fermi level are outlined by the purple (light) curves. (b) Top and side views of the charge distribution for a state near the $K_{1}$ point of (a). The yellow rectangles indicate the $A B-B A$ domain-wall structure.

amplitude of the state slightly above the $K_{1}$ point. The top view shows the extended tilt boundary in which the mismatch of one interatomic spacing is spread over a 3.1-nm distance. The side view shows that the charge for the states near $K_{1}$ is highly localized on the layer subject to compressive strain, regardless of the direction of the applied electric field. The situation for the states in the vicinity of $K_{2}$ is similar, except that the charge prefers to be highly localized on the layer under tensile strain. Hence, forming a layerselective contact to an isolated edge state could be a mechanism for valley filtering [12,23].

Finally, we comment on the so-far ignored effect of interaction. If the edge boundaries have substantial width, the forward-scattering part of the Coulomb interaction will be the dominant correlation effect to the edge states and lead to Luttinger liquid behavior [24].

\section{TILT-BOUNDARY EDGE STATES AND $\mathbb{Z}$ SPT}

In order to address the robustness of the edge states, we investigate topological aspects of the low-energy effective theory in the continuum limit. We first show that the $A B-B A$ tilt boundary can be mapped to a gate-polarity boundary of a uniform bilayer. Based on this mapping and the results of Refs. [13,20] on the gate-polarity boundary, we discuss the valley Chern number of the tiltboundary edge states. We then apply the notion of SPT [17] and identify chirally stacked multilayer graphene as a realization of $\mathbb{Z}$-type SPT, protected by time reversal $(T)$, the absence of valley mixing, and charge-conservation symmetries. This identification enables us to address the robustness of tilt-boundary edge states as well as the effects of symmetry changes: topological quantum phase transitions. There are recent studies of such perturbations for specific cases such as Rashba spin-orbit coupling [25] and magnetic ordering [26]. Through our first application of the SPT classification scheme by Wen [17] to a concrete physical system of multilayer graphene, we obtain an exhaustive systematic study of topological quantum phase-transition possibilities.

The low-energy effective Hamiltonian near the $K$ valley for a uniformly $A B$ - or $B A$-stacked bilayer is

$$
\begin{aligned}
H_{K}^{A B-B A}= & v_{F} k_{x}\left(\mu_{x} \otimes \lambda_{0}\right)+v_{F} k_{y}\left(\mu_{y} \otimes \lambda_{0}\right) \\
& -(\Delta / 2)\left(\mu_{0} \otimes \lambda_{z}\right)+\frac{t_{\perp}}{2}\left(\mu_{x} \otimes \lambda_{x}\right) \mp \frac{t_{\perp}}{2}\left(\mu_{y} \otimes \lambda_{y}\right),
\end{aligned}
$$

where a - or + sign should be used for $A B$ or $B A$ stacking, respectively. In Eq. (2), $v_{F}=3 t a / 2, \mu_{i}$ 's are Pauli matrices acting on the sublattice indices, and $\lambda_{i}$ 's are Pauli matrices acting on the layer indices. The effective Hamiltonian near $K^{\prime}$ is $H_{K^{\prime}}\left(k_{x}, k_{y}\right)=H_{K}\left(-k_{x}, k_{y}\right)$. Now, it is straightforward to show that $B A$ stacking is equivalent to $A B$ stacking, subject to the opposite gate polarity. At zero field, the Hamiltonian of the $A B$-stacked bilayer can be transformed to that of the $B A$ stacking by interchanging the two layers via the following unitary transformation: $H \rightarrow S^{\dagger} H S$, with $S=\mu_{0} \otimes \lambda_{x}$. For a gated bilayer, however, the gate polarity has to flip, since

$$
\mu_{0} \otimes \lambda_{x} H_{K}^{A B}(\Delta) \mu_{0} \otimes \lambda_{x}=H_{K}^{B A}(-\Delta) .
$$

Hence, at the level of low-energy effective theory, the tilt boundary between $A B$ and $B A$ stacking under a uniform external field is equivalent to the gate-polarity domain wall of the structurally uniform bilayer proposed by Martin et al. [13]

The above equivalence, combined with earlier results on the valley Chern number of a gated chirally stacked multilayer, offers the topological origin of the helical edge states observed in the microscopic calculation of Sec. II. First for bilayer and then for general $N$ layers, it was shown that the low-energy effective theory of a chirally stacked $N$ layer under a uniform vertical electric field $\Delta$ has a finite Chern number per spin for each valley of equal magnitude and opposite sign $[13,20,27,28]$ :

$$
C_{K}=-C_{K^{\prime}}=\frac{N}{2} \operatorname{sgn}\left(t_{\perp} \Delta\right)
$$

The Chern numbers in Eq. (4) can be obtained by integrating the Berry curvature over momenta $\left(k_{x}, k_{y}\right)$, continuing the linearized dispersion to infinity. The combination of this Chern number for chirally stacked graphene and the equivalence relation of Eq. (3) means that the valley Chern numbers change sign at the tilt boundary. Such a sign change leads to $\Delta C_{K}=-\Delta C_{K^{\prime}}=N \operatorname{sgn}\left(t_{\perp} \Delta\right)$ across the 
tilt boundary and $N$ branches of valley helical edge states [20], as long as the two valleys $K$ and $K^{\prime}$ remain distinct. Hence, the two valley helical edge states per spin observed in Sec. II originate from the valley Chern number change across the tilt boundary, as in the gate-polarity boundary edge states $[13,20]$. Hence, our prediction is that the tilt boundaries will be the first experimentally observed crystalline topological defects to host a topological gapless mode because of a change in the Chern number.

We now apply the procedure for identifying the class of SPT based on symmetries of the free fermion Hamiltonian developed by Wen [17], which predicts the possible number of protected edge (surface) states. This procedure allows us to consider additional symmetries in the multilayer graphene in addition to the $C, T$, and $P$ taken into account in the pioneering work by Schnyder et al. [16] and by Kitaev [29]. Further, the resulting classification reveals the fate of edge states upon symmetry changes.

Additional symmetries relevant for the SPT analysis of tilt-boundary edge states are (1) an antiunitary discrete symmetry operation combining time reversal and no-valley mixing $\Theta$, (2) the conservation of charge difference between two valleys $U(1)_{v}$, and (3) the conservation of total charge $U(1)_{c}$. Applying the scheme of Ref. [17], we find the chirally stacked graphene multilayer to be a $\mathbb{Z}$-type SPT (see the Appendix) showing QVH when all three symmetries are present. As gapless edge states are guaranteed at the physical boundary between the two systems with a different index $\mathbb{Z}$ such as $A B$ and $B A$ stacking, the tilt-boundary edge states are protected as long as the three symmetries are maintained.

On the other hand, a symmetry change can either yield a trivial phase that does not support an edge state or a different type of SPT with a different type of edge state. In all cases, the bulk gap has to close and reopen upon the transition between different phases. The SPT analysis without (Table I) and with (Table II) spin as a dynamic degree of freedom given in the Appendix shows that ruining the $U(1)_{v}$ symmetry through intervalley scattering is the only

TABLE I. Classification of the SPT on multilayer graphene by considering the presence $(\checkmark)$ or absence $(X)$ of the time-reversal symmetry $\hat{\Theta} \propto i \tau_{y}$, valley-polarization conservation $U(1)_{v}$, and charge conservation $U(1)_{c}$. Four upper rows classify topological insulators, while four lower rows classify TSCs.

\begin{tabular}{ccccc}
\hline \hline$\Theta$ & $\mathrm{U}(1)_{v}$ & $U(1)_{c}$ & Classification & Examples \\
\hline$\checkmark$ & $\checkmark$ & $\checkmark$ & $\mathbb{Z}$ & QVH \\
$\times$ & $\checkmark$ & $\checkmark$ & $\mathbb{Z} \oplus \mathbb{Z}$ & Intravalley QAH \\
$\checkmark$ & $\times$ & $\checkmark$ & Trivial & Trivial insulator \\
$\times$ & $\times$ & $\checkmark$ & $\mathbb{Z}$ & Intervalley QAH \\
$\checkmark$ & $\checkmark$ & $\times$ & $\mathbb{Z}$ & TVSC \\
$\times$ & $\checkmark$ & $\times$ & $\mathbb{Z} \oplus \mathbb{Z}$ & Intravalley TSC \\
$\checkmark$ & $\times$ & $\times$ & Trivial & Trivial superconductor \\
$\times$ & $\times$ & $\times$ & $\mathbb{Z}$ & Intervalley TSC \\
\hline \hline
\end{tabular}

TABLE II. Classification of spinful SPT insulators on multilayer graphene. Three kinds of time-reversal operators are considered for classification because of the valley and spin dynamical degrees of freedom: $\hat{\Theta}_{1} \propto \tau_{x}$ exchanges two valleys; $\hat{\Theta}_{2} \propto i \sigma_{y}$, which acts on the spin indices, flips spin; and $\hat{T}=$ $\hat{\Theta}_{1} \hat{\Theta}_{2}$ does both. ${ }^{\text {a }}$ The upper three rows show that the classification reduces to that of a spinless fermion if $\hat{\Theta}_{2}$ symmetry is absent. The lower rows show new possibilities that emerge upon taking spins into account.

\begin{tabular}{cccccc}
\hline \hline$\Theta_{1}$ & $\Theta_{2}$ & $T=\Theta_{1} \Theta_{2}$ & $\mathrm{U}(1)_{v}$ & Classification & Examples \\
\hline$\checkmark$ & $\times$ & $\times$ & $\checkmark$ & $\mathbb{Z}$ & QVH \\
$\times$ & $\times$ & $\times$ & $\checkmark$ & $\mathbb{Z} \oplus \mathbb{Z}$ & Intravalley QAH \\
$\times$ & $\times$ & $\times$ & $\times$ & $\mathbb{Z}$ & Intervalley QAH \\
$\checkmark$ & $\checkmark$ & $\checkmark$ & $\checkmark$ & $\mathbb{Z}_{2}$ & LAF \\
$\times$ & $\checkmark$ & $\times$ & $\checkmark$ & $\mathbb{Z}_{2} \mathbb{Z}_{2}$ & Intravalley topological \\
insulator \\
$\times$ & $\checkmark$ & $\times$ & $\times$ & $\mathbb{Z}_{2}$ & Intervalley topological \\
insulator \\
$\times$ & $\times$ & $\checkmark$ & $\checkmark$ & $\mathbb{Z}$ & Intravalley QSH \\
\hline \hline
\end{tabular}

${ }^{\mathrm{a}}$ The classification for the superconductors is identical to that for the insulators.

way to arrive at a trivial phase without edge states. However, such a large momentum transfer generally requires fine-tuning unless the unit cell becomes enlarged either for the entire system [30,31] or for short-range disorder, such as a vacancy-breaking $A-B$ sublattice symmetry [32]. As both rarely occur, we anticipate gapless edge states at most tilt boundaries except for armchair boundaries.

Among symmetry-change possibilities leading to another SPT, the transition from QVH with spin degeneracy to the layer antiferromagnet (LAF) phase where spin is a dynamic degree of freedom is of particular interest, as LAF is suspected to be the ground state of bilayer graphene near the neutrality point [33]. Upon this phase transition, the nature of the edge states changes from spin-degenerate valley helical states $(\mathrm{QVH})$ to spin-valley Hall edge states (LAF). In such transitions, the bulk gap has to close and reopen; this is indeed seen in the experiment of Velasco et al. [33].

\section{CONNECTION TO EXPERIMENTS}

In this section, we discuss the experimental implications of our findings. Specifically, we propose that transport through the network of tilt boundaries may resolve the long-standing mystery of subgap transport [1,5]. Further, we propose feasible experiments to test the proposal.

In order to discuss the topological transport through the network of tilt boundaries observed in Refs. [6-8], we should first discuss the effect of the arbitrary angle between the Burger's vector $\vec{b}$ and the tangent vector $\vec{t}$. Microscopic study of tilt boundaries at various angles between $\vec{b}$ and $\vec{t}$ will be presented in the future [34]. However, it has been 
known that a single-domain Bernal-stacked gapped bilayer ribbon should support a gapless edge state for zigzag edges but not for armchair edges [20,35]. The edge state gaps out for armchair edges because the armchair edge enlarges the unit cell along the direction parallel to the boundary and makes the projection of the $K$ and $K^{\prime}$ valleys identical. However, Jung et al. [20] showed that the polarity boundary edge states only develop a barely visible gap that is orders of magnitude less then the bulk gap, even for a sharp boundary, and the gap decreases quickly when the polarity boundary becomes smooth. These arguments apply to our tilt boundary, and the edge state will develop a small gap when $\vec{b} \| \vec{t}$. However, given the large width of the observed tilt boundaries, we expect that all straight tilt boundaries will have nearly gapless edge states, except those with small angles between $\vec{b}$ and $\vec{t}$.

When the tilt boundary meanders and changes directions, there will likely be portions with small gaps segmenting the gapless regions, and the transport will occur through hopping between the gapless regions. The observed 2D network of such tilt boundaries would yield a 2D variable-range-hopping temperature dependence $R(T) \propto \exp \left(T_{0} / T\right)^{1 / 3}[36,37]$ at low temperatures, governed by the $2 \mathrm{D}$ connectivity and the small characteristic gaps of gapped regions. Such $\exp \left(T_{0} / T\right)^{1 / 3}$ dependence of resistivity is consistent with the observed temperature $T$ dependence of resistance at low temperatures $[1,5]$.

We propose the following experiments to test our proposal. (1) Four terminal transport measurements with two of the contacts, say, contacts 1 and 3 , at two ends of a tilt boundary. This experiment would yield highly anisotropic transport, proving dominant transport along the tilt boundary, i.e., $R_{1,3} \ll R_{2,4}$. (2) Scanning-tunneling-spectroscopy measurements of the local density of states. This experiment should measure a gapless spectrum at the tilt boundary but exhibit a gapped spectrum with the gap magnitude of the optical gap away from the tilt boundary. (3) Thermoelectric imaging. The midgap density of states at tilt boundaries would appear in scanning thermopower images. Unpublished thermopower-imaging data by Cho et al. [38] indeed show a network with a local density of states near Fermi energy that is reminiscent of the tiltboundary network. (4) Edge-current imaging using scanning SQUID that can detect the magnetic field generated by edge currents.

\section{CONCLUSION}

We showed that spin-degenerate tilt boundaries of gated multilayer graphene support topological gapless edge states protected by three symmetries: time (valley) reversal, no-valley mixing, and electron-number conservation. We demonstrated the existence of gapless edge states through a tight-binding model calculation and a firstprinciples calculation, where the latter calculation took strain effects into account. We then addressed the symmetry protection of the edge states and the consequences of symmetry changes within the framework of the SPT [17].

The framework of the SPT allowed us to place the 2D topological phase supporting the edge states, namely, QVH, among various topological-insulator or superconductor phases, alongside previously postulated quantum anomalous Hall (QAH), LAF, and quantum spin Hall (QSH) phases. While previous literature postulated QVH, QAH, LAF, and QSH to be all supporting a number of edge states growing with the number of layers $N$ (i.e., $\mathbb{Z}$ type in the language of classification), we found that the symmetry of LAF only protects an odd number of edge modes for each valley. Hence, $L A F$ is a $\mathbb{Z}_{2}$ topological insulator, much like a quantum spin Hall insulator [39]. Transitions between these different SPTs require closing and reopening of the bulk gap, as has already been observed in Ref. [33].

We predict the naturally occurring tilt boundary [6-8] to be the first topological structural defect that hosts a topologically protected gapless mode of transport. Most importantly, our findings on tilt boundaries combined with the recent observations [6-8] may explain the long-standing mystery of subgap transport [1]. Our prediction of the topological kink states at the tilt boundary can be tested through proposed transport, scanning-tunneling spectroscopy, thermopower-imaging experiments, and scanning SQUID experiments. Experimental confirmation of the tilt-boundary transport origin of the subgap transport will open doors to control the subgap transport and enable the device application of gated multilayer graphene systems.

\section{ACKNOWLEDGMENTS}

We thank Paul McEuen for numerous discussions and sharing his unpublished data. We thank Joe Stroscio for sharing his unpublished data on thermopower imaging. We thank E. J. Mele for useful discussions and sharing the preprint [45] and J. Sethna for useful discussions regarding the structural topological defect aspect of the tilt boundary. E.-A. K. and D. N. were supported by NSF Grant No. EEC0646547 through Cornell Center for Nanoscience. E.-A. K. and A. V. were supported in part by NSF CAREER Grant No. DMR-0955822. E.-A. K. was also supported in part by NSF Grant No. DMR-1120296 through Cornell Center for Materials Research. Y. L. and L. Y. were supported by NSF Grant No. DMR-1207141. The computational resources have been provided by Lonestar of Teragrid at the Texas Advanced Computing Center (TACC).

Note added.-Recently, a complementary preprint [45], which covers closely related material, has appeared.

\section{APPENDIX: CHIRAL MULTILAYER GRAPHENE AS $\mathbb{Z}$-TYPE SPT}

The procedure consists of three steps. (1) Find a gapless Dirac Hamiltonian (by keeping the kinetic term only) with 
the same symmetries. Then, we find all the symmetrypreserving mass terms that can gap out the gapless part and are amenable to classification using Clifford algebra. This procedure is based on the assumption that the SPT order is robust as long as the energy gap stays finite and the symmetries remain the same, and hence any gapped Hamiltonian can be adiabatically transformed into a gapped Dirac Hamiltonian. (2) Express the Hamiltonian and the conserved quantities associated with symmetries in the Majorana basis. This step leads to the Clifford algebra (i.e., real representation of the Dirac algebra) associated with the gapless part of the Hamiltonian. (3) Find the space of mass matrices that anticommutes with all the generators of this Clifford algebra. The resulting space may have disconnected pieces, the number of which gives the classification of the SPT. Two mass matrices are topologically distinct if and only if they belong to two different pieces. Applying this procedure to chiral multilayer graphene will enable us to study phase transitions into different SPTs upon symmetry changes.

For a chiral multilayer graphene system, we assume time-reversal symmetry $(T)$ and no-valley mixing, which can be combined to form an antiunitary operator $\Theta$. In addition, we assume conservation of valley polarization $\left[U(1)_{v}\right]$ and total charge $\left[U(1)_{c}\right]$. The relevant gapless Dirac Hamiltonian is

$$
H=i v_{F} \int d^{2} x \Psi^{\dagger}(x)\left(\rho_{1} \partial_{x}+\rho_{2} \partial_{y}\right) \Psi(x),
$$

where $\quad \Psi^{T}=\left(c_{A, K}, c_{A, K^{\prime}}, c_{B, K}, c_{B, K^{\prime}}\right), \quad \rho_{1}=\mu_{x} \otimes \tau_{z} \otimes$ $I_{n \times n}$, and $\rho_{2}=\mu_{y} \otimes \tau_{0} \otimes I_{n \times n}$, in which $n$ is given by the number of layers. This Hamiltonian can be written in the Majorana fermion basis using the following decomposition:

$$
c_{\mu, \tau}(x)=\frac{\gamma_{+, \mu, \tau}(x)+i \gamma_{-, \mu, \tau}(x)}{2},
$$

where $\gamma$ denotes the Majorana fermion satisfying

$$
\gamma_{\alpha, \mu, \tau}^{2}=1, \quad\left\{\gamma_{+, \mu, \tau}, \gamma_{-, \mu, \tau}\right\}=0,
$$

where $\mu(\tau)$ denotes the $A$ or $B$ sublattice ( $K$ or $K^{\prime}$ valley) indices, and $\alpha$ denotes the flavor of the Majorana fermions $(+$ or -$)$. In the Majorana fermion basis, the Hamiltonian is represented as follows:

$$
H=i \int d^{2} x \eta(x) \mathcal{A} \eta(x),
$$

where $\mathcal{A}$ is a real antisymmetric matrix (differential operator), and $\eta$ is an eight-component vector whose components are $\gamma_{\alpha, \mu, \tau}$.

Now, we express the conserved quantities associated with the symmetries of the Hamiltonian in the Majorana fermion basis. First, no-valley mixing combined with total electron-number-conservation symmetry leads to separate conservation of the electron number at each valley $N_{K}$ and
$N_{K^{\prime}}$. Hence, the total electron number $N_{c}=N_{K}+N_{K^{\prime}}$ and the valley polarization $N_{V}=N_{K}-N_{K^{\prime}}$ are conserved. In the Majorana fermion basis,

$$
\begin{aligned}
N_{c, V} & =\frac{i}{4} \int d^{2} x \eta(x) \hat{Q}_{c, V} \eta(x), \quad \text { with } \\
\hat{Q}_{c} & =i \alpha_{y} \otimes \mu_{0} \otimes \tau_{0} \otimes I, \\
\hat{Q}_{V} & =i \alpha_{y} \otimes \mu_{0} \otimes \tau_{z} \otimes I,
\end{aligned}
$$

where $\alpha_{i}\left(\tau_{i}\right)$ Pauli matrices act on the Majorana flavors (valley indices). So defined, $Q_{c}$ and $Q_{V}$ satisfy $\hat{Q}_{c}^{2}=$ $\hat{Q}_{V}^{2}=-1$.

Under time-reversal symmetry $T, K$ and $K^{\prime}$ valley indices are exchanged; i.e., $\hat{T}: c_{\mu, K} \leftrightarrow c_{\mu, K^{\prime}}$. Hence, $T$ acts like $\tau_{x}$ in the valley basis, with the matrix part of the timereversal operator satisfying $\hat{T}^{2}=1$. On the other hand, novalley mixing implies that the Hamiltonian is invariant under $\left(c_{\mu, K}, c_{\mu, K^{\prime}}\right) \rightarrow\left(c_{\mu, K},-c_{\mu, K^{\prime}}\right)$ transformation, which acts like $\tau_{z}$ in the valley basis. Hence, in the presence of no-valley-mixing symmetry, we can define a new time-reversal operator $\hat{\Theta}:\left(c_{\mu, K}, c_{\mu, K^{\prime}}\right) \rightarrow\left(c_{\mu, K^{\prime}},-c_{\mu, K}\right)$, which acts like $\tau_{z} \hat{T}=i \tau_{y}$. In terms of Majorana fermions,

$$
\hat{\Theta}=\alpha_{0} \otimes \mu_{0} \otimes\left(i \tau_{y}\right) \otimes I, \quad \hat{\Theta}^{2}=-1 .
$$

In order to find the relevant Clifford algebra, we need to form anticommuting generators in terms of $\mathcal{A}$ in Eq. (A4) combined with symmetries $\hat{Q}_{c}, \hat{Q}_{V}$, and $\hat{\Theta}$. However, the symmetries require $\left[\mathcal{A}, \hat{Q}_{c}\right]=\left[\mathcal{A}, \hat{Q}_{V}\right]=0$ and $\{\mathcal{A}, \hat{\Theta}\}=0$. Moreover, symmetry operators satisfy $\hat{Q}_{c} \hat{\Theta}=\hat{\Theta} \hat{Q}_{c}, \hat{Q}_{V} \hat{\Theta}=-\hat{\Theta} \hat{Q}_{V}$, and $\hat{Q}_{c} \hat{Q}_{V}=\hat{Q}_{V} \hat{Q}_{c}$ relations. Using these relations, we find the full set of generators of the relevant Clifford algebra as $\rho_{1}, \rho_{2}$, and

$$
\rho_{3}=\hat{\Theta} \hat{Q}_{V} \hat{Q}_{c}, \quad \rho_{4}=\hat{\Theta}, \quad \rho_{5}=\hat{\Theta} \hat{Q}_{V},
$$

as $\left\{\mathcal{A}, \rho_{i}\right\}=0$ for $i=3,4,5$. The resulting full set of anticommutation relations is

$$
\left\{\rho_{i}, \rho_{j}\right\}=2 g_{i, j}, \quad g_{i, j}=\operatorname{diag}(1,1,1,-1,-1),
$$

and it defines a Clifford algebra $\operatorname{Cliff}(3,2)$.

Now, we will find the space of mass matrices $C_{M}$ that can gap out the Dirac Hamiltonian associated with this Clifford algebra in order to obtain SPT classification. The mass term with matrix representation $H_{M}=$ $\frac{i}{4} \sum_{I, J} M_{I, J} \eta_{I} \eta_{J}$ should satisfy the following algebra:

$$
M \rho_{i}=-\rho_{i} M, \quad M^{2}=-1,
$$

where we have normalized $M$. Solving the above equation yields the allowed space for the mass matrix $C_{M}$. It has been shown [17] that $C_{M}$, which solves Eq. (A9) for the case of $\operatorname{Cliff}(3,2)$, is

$$
C_{M}=\lim _{n \rightarrow \infty} \bigcup_{m=0}^{n} \frac{O(n)}{O(m) \times O(n-m)} .
$$


The SPT classification is then given by the number of disconnected pieces in the space of mass matrix $C_{M}$, i.e., its zeroth homotopy group: $\pi_{0}\left(C_{M}\right)$. Using Eq. (A10), it can be verified that $\pi_{0}\left(C_{M}\right)=\mathbb{Z}$ [17]. Consequently, each class of the time-reversal-invariant multilayer graphene in the absence of intervalley scattering is indexed by a $\mathbb{Z}$-valued number, in this case, the valley Chern number.

Now, we are in a good position to consider symmetry changes. It is important to note here that spontaneously ordered phases can be considered alongside systems under an external field, as once a system is deep inside the ordered phase, it can be treated within mean-field theory.

We first consider the symmetry-reduction possibilities while maintaining spin degeneracy (see Table I). If we only break the time-reversal symmetry, the system is characterized by two independent topological indices $\left(C_{K}, C_{K^{\prime}}\right)$; hence, the classification is given by $\mathbb{Z} \oplus \mathbb{Z}$. We refer to these SPT phases as intravalley QAH states. Such phases may be realized by placing trigonally strained graphene $[40,41]$ under an external magnetic field, as the sign of the pseudomagnetic field caused by strain is opposite for the two valleys. Further reducing the symmetry by introducing intervalley scattering leads to an intervalley QAH state indexed by a single integer $\mathbb{Z}$ : the total Chern number [42]. Breaking electron-number conservation turns the above insulators into superconductors. Following the procedure above, we obtain the same classification for the TSCs in 2D, resulting in a topological valley superconductor (TVSC) and intra- or intervalley TSCs. Table I summarizes all symmetry-reduction possibilities and their classifications, starting from gated multilayer graphene.

Now, we consider extending our classification to take the electron spin into account as a dynamical degree of freedom. This generalization will allow us to consider interaction effects at the level of spin ordering. With both spin and valley degrees of freedom, there are three symmetry operators related to time reversal: $\hat{\Theta}_{1} \propto \tau_{x}$ exchanges two valleys; $\hat{\Theta}_{2} \propto i \sigma_{y}$, which acts on the spin indices, flips spin; and $\hat{T}=\hat{\Theta}_{1} \hat{\Theta}_{2}$ does both. If both $\hat{\Theta}_{2}$ and $\hat{T}$ are broken, the classification reduces to that of spinless electrons (see the upper part of Table II). However, taking any of these symmetry operators into account leads to new classes (see the lower part of Table II).

When all $\hat{\Theta}_{1}, \hat{\Theta}_{2}$ (and as a result $\hat{T}$ ), and no-valleymixing symmetries are imposed, the state corresponds to the so-called LAF phase predicted in Refs. $[28,43]$ and possibly occurring as a ground state in bilayer graphene at the neutrality point [33]. In this phase, the product of the spin and valley of edge quasiparticles is locked to their momentum. Therefore, one may index this state by its spinvalley Chern number $C_{\mathrm{SV}}$ [28]. In this phase, for each valley, the edge states associated with two spins are counterpropagating. As long as these two counterpropagating modes do not couple, there can be any number of them per valley, leading to $2 C_{\mathrm{Sv}} e^{2} / h$ spin-valley Hall conductivity
$[12,28]$. However, there is a form of symmetry-allowed coupling between a pair of sets of counterpropagating edges; this coupling can gap out the edge modes [39]. Therefore, the number of symmetry-protected edge modes for each valley is $C_{\mathrm{SV}} \bmod 2$. Hence, we obtain a $\mathbb{Z}_{2}$ classification for this phase labeled by $(-1)^{C_{\mathrm{Sv}}}$, as opposed to $\mathbb{Z}$ classification, which would be implied by Refs. [12,28].

Another interesting possibility is breaking $\hat{\Theta}_{1}$ and $\hat{\Theta}_{2}$ while respecting their product $\hat{T}$ and no-valley-mixing symmetry. This leads to the QSH phase, in which $C_{K, \uparrow}=$ $-C_{K^{\prime}, \downarrow}$ and $C_{K^{\prime}, \uparrow}=-C_{K, \downarrow}$ because of $\hat{T}$ symmetry, while there is no constraint on the $C_{K, \uparrow}+C_{K, \downarrow}$. Hence, unlike the usual 2D QSH states $[14,15]$, we obtain $\mathbb{Z}$. This classification is because of the multiple types of time-reversal operators that can be defined [44] in the presence of novalley-mixing symmetry. This and other SPT possibilities are summarized in Table II.

[1] Jeroen B. Oostinga, Hubert B. Heersche, Xinglan Liu, Alberto F. Morpurgo, and Lieven M. K. Vandersypen, Gate-Induced Insulating State in Bilayer Graphene Devices, Nat. Mater. 7, 151 (2008).

[2] Edward McCann, Asymmetry Gap in the Electronic Band Structure of Bilayer Graphene, Phys. Rev. B 74, 161403(R) (2006).

[3] Yuanbo Zhang, Tsung-Ta Tang, Caglar Girit, Zhao Hao, Michael C. Martin, Alex Zettl, Michael F. Crommie, Y. Ron Shen, and Feng Wang, Direct Observation of a Widely Tunable Bandgap in Bilayer Graphene, Nature (London) 459, 820 (2009).

[4] Jian Li, Ivar Martin, Markus Büttiker, and Alberto F. Morpurgo, Topological Origin of Subgap Conductance in Insulating Bilayer Graphene, Nat. Phys. 7, 38 (2011).

[5] Jun Yan and Michael S. Fuhrer, Charge Transport in Dual Gated Bilayer Graphene with Corbino Geometry, Nano Lett. 10, 4521 (2010).

[6] Jonathan Alden, Adam W. Tsen, Pinshane Y. Huang, Robert Hovden, Lola Brown, Jiwoong Park, David A. Muller, and Paul L. McEuen, Interlayer Strain Solitons in Bilayer Graphene (to be published).

[7] S. Hattendorf, A. Georgi, V. Geringer, M. Liebmann, and M. Morgenstern, Graphene on Mica Probed by Scanning Tunneling Microscopy: Networks of $A B A$ and $A B C$ Stacked Graphene and Corrugation of the Monolayer, arXiv:1207.5427.

[8] Lola Brown, Robert Hovden, Pinshane Huang, Michal Wojcik, David A. Muller, and Jiwoong Park, Twinning and Twisting of Tri- and Bilayer Graphene, Nano Lett. 12, 1609 (2012).

[9] Ying Ran, Yi Zhang, and Ashvin Vishwanath, OneDimensional Topologically Protected Modes in Topological Insulators with Lattice Dislocations, Nat. Phys. 5, 298 (2009).

[10] Maissam Barkeshli and Xiao-Liang Qi, Topological Nematic States and Non-Abelian Lattice Dislocations, Phys. Rev. X 2, 031013 (2012). 
[11] Jeffrey C. Y. Teo and C. L. Kane, Topological Defects and Gapless Modes in Insulators and Superconductors, Phys. Rev. B 82, 115120 (2010).

[12] Di Xiao, Wang Yao, and Qian Niu, Valley-Contrasting Physics in Graphene: Magnetic Moment and Topological Transport, Phys. Rev. Lett. 99, 236809 (2007).

[13] Ivar Martin, Ya. M. Blanter, and A.F. Morpurgo, Topological Confinement in Bilayer Graphene, Phys. Rev. Lett. 100, 036804 (2008).

[14] C. L. Kane and E. J. Mele, Quantum Spin Hall Effect in Graphene, Phys. Rev. Lett. 95, 226801 (2005).

[15] B. Andrei Bernevig and Shou-Cheng Zhang, Quantum Spin Hall Effect, Phys. Rev. Lett. 96, 106802 (2006).

[16] Andreas P. Schnyder, Shinsei Ryu, Akira Furusaki, and Andreas W. W. Ludwig, Classification of Topological Insulators and Superconductors in Three Spatial Dimensions, Phys. Rev. B 78, 195125 (2008).

[17] Xiao-Gang Wen, Symmetry-Protected Topological Phases in Noninteracting Fermion Systems, Phys. Rev. B 85, 085103 (2012).

[18] Liang Fu, Topological Crystalline Insulators, Phys. Rev. Lett. 106, 106802 (2011).

[19] P. Dziawa, B. J. Kowalski, K. Dybko, R. Buczko, A. Szczerbakow, M. Szot, E. Łusakowska, T. Balasubramanian, B.M. Wojek, M.H. Berntsen, O. Tjernberg, and T. Story, Topological Crystalline Insulator States in $\mathrm{Pb}_{1-x} \mathrm{Sn}_{x} \mathrm{Se}$, Nat. Mater. 11, 1023 (2012).

[20] Jeil Jung, Fan Zhang, Zhenhua Qiao, and Allan H. MacDonald, Valley-Hall Kink and Edge States in Multilayer Graphene, Phys. Rev. B 84, 075418 (2011).

[21] P. Hohenberg and W. Kohn, Inhomogeneous Electron Gas, Phys. Rev. 136, B864 (1964).

[22] W. Kohn and L.J. Sham, Self-Consistent Equations Including Exchange and Correlation Effects, Phys. Rev. 140, A1133 (1965).

[23] A. Rycerz, J. Tworzydło, and C. W. J. Beenakker, Valley Filter and Valley Valve in Graphene, Nat. Phys. 3, 172 (2007).

[24] Matthew Killi, Tzu-Chieh Wei, Ian Affleck, and Arun Paramekanti, Tunable Luttinger Liquid Physics in Biased Bilayer Graphene, Phys. Rev. Lett. 104, 216406 (2010).

[25] Zhenhua Qiao, Wang-Kong Tse, Hua Jiang, Yugui Yao, and Qian Niu, Two-Dimensional Topological Insulator State and Topological Phase Transition in Bilayer Graphene, Phys. Rev. Lett. 107, 256801 (2011).

[26] Wang-Kong Tse, Zhenhua Qiao, Yugui Yao, A.H. MacDonald, and Qian Niu, Quantum Anomalous Hall Effect in Single-Layer and Bilayer Graphene, Phys. Rev. B 83, 155447 (2011).

[27] Alberto Cortijo, Adolfo G. Grushin, and María A.H. Vozmediano, Topological Insulating Phases in Monolayer and Bilayer Graphene: An Effective Action Approach, Phys. Rev. B 82, 195438 (2010).

[28] Fan Zhang, Jeil Jung, Gregory A. Fiete, Qian Niu, and Allan H. MacDonald, Spontaneous Quantum Hall States in Chirally Stacked Few-Layer Graphene Systems, Phys. Rev. Lett. 106, 156801 (2011).
[29] Alexei Kitaev, in Advances in Theoretical Physics, edited by Vladimir Lebedev and Mikhail Feigel'man, AIP Conf. Proc. No. 1134 (AIP, New York, 2009), p. 22-30.

[30] Chang-Yu Hou, Claudio Chamon, and Christopher Mudry, Electron Fractionalization in Two-Dimensional Graphenelike Structures, Phys. Rev. Lett. 98, 186809 (2007).

[31] R. Jackiw and S.-Y. Pi, Chiral Gauge Theory for Graphene, Phys. Rev. Lett. 98, 266402 (2007).

[32] Hidekatsu Suzuura and Tsuneya Ando, Crossover from Symplectic to Orthogonal Class in a Two-Dimensional Honeycomb Lattice, Phys. Rev. Lett. 89, 266603 (2002).

[33] J. Velasco, Jr., L. Jing, W. Bao, Y. Lee, P. Kratz, V. Aji, M. Bockrath, C. N. Lau, C. Varma, R. Stillwell, D. Smirnov, Fan Zhang, J. Jung, and A. H. MacDonald, Transport Spectroscopy of Symmetry-Broken Insulating States in Bilayer Graphene, Nat. Nanotechnol. 7, 156 (2012).

[34] Yufen Liang et al., Boundary Condition Dependence of the AB-BA Tilt Boundary Kink States (to be published).

[35] Eduardo V. Castro, N. M. R. Peres, J. M. B. Lopes dos Santos, A.H. Castro Neto, and F. Guinea, Localized States at Zigzag Edges of Bilayer Graphene, Phys. Rev. Lett. 100, 026802 (2008).

[36] N.F. Mott, Conduction in Glasses Containing Transition Metal Ions, J. Non-Cryst. Solids 1, 1 (1968).

[37] D. N. Tsigankov and A. L. Efros, Variable Range Hopping in Two-Dimensional Systems of Interacting Electrons, Phys. Rev. Lett. 88, 176602 (2002).

[38] Sanghee Cho, Stephen Dongmin Kang, Wondong Kim, Eui-Sup Lee, Sung-Jae Woo, Ki-Jeong Kong, Ilyou Kim, Hyeong-Do Kim, Tong Zhang, Joseph Stroscio, YongHyun Kim, and Ho-Ki Lyeo, Thermoelectric Imaging of Structural Disorder in Epitaxial Graphene (to be published).

[39] C. L. Kane and E. J. Mele, $Z_{2}$ Topological Order and the Quantum Spin Hall Effect, Phys. Rev. Lett. 95, 146802 (2005).

[40] N. Levy, S. A. Burke, K. L. Meaker, M. Panlasigui, A. Zettl, F. Guinea, A. H. Castro Neto, and M. F. Crommie, Strain-Induced Pseudo-magnetic Fields Greater than 300 Tesla in Graphene Nanobubbles, Science 329, 544 (2010).

[41] F. Guinea, M. I. Katsnelson, and A. K. Geim, Energy Gaps and a Zero-Field Quantum Hall Effect in Graphene by Strain Engineering, Nat. Phys. 6, 30 (2010).

[42] As far as symmetry of the phase is concerned, the quantum anomalous Hall state and the quantum Hall state are equivalent.

[43] Oskar Vafek, Interacting Fermions on the Honeycomb Bilayer: From Weak to Strong Coupling, Phys. Rev. B 82, 205106 (2010).

[44] Denis Bernard, Eun-Ah Kim, and André LeClair, Edge States for Topological Insulators in Two Dimensions and Their Luttinger-like Liquids, Phys. Rev. B 86, 205116 (2012).

[45] Fan Zhang, A.H. MacDonald, and E. J. Mele, Valley Chern Numbers and Boundary Modes in Gapped Bilayer Graphene, arXiv:1301.4205. 